\title{
Civilisations
}

Revue internationale d'anthropologie et de sciences

humaines

$65 \mid 2016$

Figures du malentendu

\section{Etre étonné. Lorsque le malentendu et l'angoisse nous ancrent dans le terrain}

\section{Edgar Tasia}

\section{(2) OpenEdition \\ Journals}

Édition électronique

URL : http://journals.openedition.org/civilisations/4075

DOI : 10.4000/civilisations.4075

ISSN : 2032-0442

\section{Éditeur}

Institut de sociologie de l'Université Libre de Bruxelles

\section{Édition imprimée}

Date de publication : 19 décembre 2016

Pagination : 127-144

ISBN : 2-9602017-0-3

ISSN : 0009-8140

Référence électronique

Edgar Tasia, «Etre étonné. Lorsque le malentendu et l'angoisse nous ancrent dans le terrain », Civilisations [En ligne], 65 | 2016, mis en ligne le 19 décembre 2019, consulté le 15 janvier 2021. URL : http://journals.openedition.org/civilisations/4075; DOI : https://doi.org/10.4000/civilisations.4075 


\title{
Etre étonné \\ Lorsque le malentendu et l'angoisse nous ancrent dans le terrain
}

\author{
Edgar TASIA
}

Résumé : Intrinsèquement liés, le malentendu et l'angoisse sont deux expériences auxquelles est souvent confronté l'anthropologue. Elles prennent racine dans le hiatus interactionnel de la rencontre, dans cette confrontation avec l'Autre dont on ne connaît pas (encore) grand-chose. L'observation, la participation, la communication, tous ces outils dont se sert l'anthropologue pour parvenir à faire son travail, sont autant de portes ouvertes au malentendu et à l'angoisse. Sur le terrain, l'anthropologue est donc rendu vulnérable aux complications communicationnelles et affectives. Mais, cette condition épistémique " fragile 》 - condition qu'il s'agira d'étudier plus en détail et d'illustrer par des vignettes tirées de mon terrain ethnographiqueest-elle vraiment "malheureuse " ? J'aimerais, dans ce texte, le démentir. En effet, si le malentendu et l'angoisse peuvent poser problème, ils peuvent également jouer un rôle pratique important pour le chercheur en aiguisant ses capacités cognitivo-affectives et en lui permettant d'éduquer son attention, de prolonger l'effort de la concentration et, in fine, d'être plus alerte aux détails et conscient des subtilités de la vie sociale. Dérouté, confus, mal à l'aise, l'anthropologue doit être extrêmement attentif à la situation dans laquelle il est englué pour parvenir à la comprendre et réduire l'angoisse qu'elle engendre.

Mots-clés : malentendu, angoisse, terrain, éducation de l'attention, méthodologie.

Abstract: Misunderstanding and anxiety are two related experiences that the anthropologist often encounters during fieldwork. They take root in the interactional hiatus of the encounter: when the anthropologist meets the Other about whom (s)he does not know much. The methodological tools used by the anthropologist (such as observing, participating and communicating) are potential occasions for misunderstanding and anxiety. Thus, on the field, the anthropologist becomes vulnerable to communicational and emotional issues. However, is this "fragile" epistemological condition really "unfortunate"? I question that by using excerpts from my own ethnographic fieldwork. Indeed, if misunderstanding and anxiety can be problematic, they can also play an important practical role for the researcher. They allow to be more conscious of interactional details and subtleties of social life by sharpening one's cognitive and affective abilities, by educating one's attention and by extending the effort of concentration. Hence, a confused and queasy anthropologist must be extremely attentive to the situation in which (s)he is stuck in order to understand and reduce the anxiety that it entails.

Keywords: misunderstanding, anxiety, fieldwork, education of attention, methodology. 


\section{Introduction}

«Qu'il faut, au moins, un sujet».

Jeanne Favret-Saada, Les mots, la mort, les sorts.

Soit un ethnographe : il a choisi d'enquêter sur le traumatisme colonial des Aborigènes d'Australie dans la banlieue de Sydney. Il n'a que très peu d'expérience de terrain puisqu'il vient juste d'entamer sa thèse de doctorat au sein d'une université francophone de Belgique. Il part donc au bout du monde pendant un mois et demi afin de faire un premier terrain exploratoire. Méticuleusement, il s'est préparé : il a parcouru de long en large l'abondante littérature sur le sujet, il a décortiqué de nombreux manuels d'enquête et en a extrait les injonctions qui lui semblaient les plus pertinentes pour réaliser son travail une fois sur place. Notons encore qu'il est belge, a un bon niveau d'anglais et qu'il est en bonne santé physique. Ayant réalisé son mémoire de maîtrise sur l'impact émotionnel de l'enquête de terrain sur l'anthropologue (plus particulièrement, sur les angoisses que ressent ce dernier), il se sent particulièrement averti des difficultés affectives que peut entraîner la pratique ethnographique ; il s'agit dorénavant pour lui de les expérimenter in vivo et non plus seulement intellectuellement. Il se rassure et se dit qu'au fond, il est bien préparé, que rien ne peut lui arriver, ni véritablement le surprendre puisqu'il s'attend à être surpris par l'expérience.

Bien sûr, il a tort ; au même titre que l'expérience d'enchantement, cette « promesse de surprise » subtilement décrite par Emmanuel Belin (2002 : 226), la pratique de l'anthropologie repose sur un paradoxe : « on s'attendait à être surpris, mais pourtant cela nous surprend » (Belin, 2002 : 226). Mais, contrairement aux expériences dont fait état le sociologue précité, les surprises du terrain ne sont pas toujours agréables ni enchanteresses. Ou, plutôt, elles ne sont pas toujours des plus faciles à vivre.

Parmi ces surprises ethnographiques, deux sont particulièrement riches d'ambiguité : le malentendu et l'angoisse que celui-ci engendre. Si, lors de la « prise » par le terrain stricto sensu, elles semblent placer le chercheur dans une situation difficile, celui-ci constatera lors de la « reprise », qu' à la réflexion, elles lui ont appris beaucoup sur luimême ainsi que sur le mode d'être au monde des individus qu'il est venu rencontrer' ${ }^{1}$. En effet, ainsi « embarrassé » par le malentendu, s'il veut comprendre quelque chose à la situation dans laquelle il se trouve et réduire l'angoisse qui l'étreint, l'ethnographe doit être particulièrement attentif à ce qui l'entoure. Son attention sera alors plus acérée et sa sensibilité affective exacerbée. L'ambiguïté de la situation provoque certes un malaise mais elle multiplie également ses capacités cognitives et affectives et lui permet d'observer et de comprendre avec plus d'acuité ce qui l'entoure. Expériences ethnographiques difficiles donc, mais heuristiquement fécondes.

Avant de nous emparer de cette problématique et de la traiter plus en profondeur, suivons, pour un instant, notre jeune ethnographe sur son terrain australien.

1 J'emprunte ici les termes de « prise » et de « reprise » à Jeanne Favret-Saada qui a brillamment théorisé ces deux dimensions de la recherche anthropologique (Favret-Saada, 1977 ; Favret-Saada, 2009). 


\section{Vignette ethnographique I}

Soit un ethnographe, donc. Au cours du mois de mai 2015, il part pour l'Australie dans le but d'y effectuer un terrain exploratoire pour ses recherches. Après plusieurs semaines d'errance peu productive, de balbutiements ethnographiques, d'observations rapides et de prises de notes à la volée, il prend contact avec une personne ressource, $\mathrm{K}$, un Aborigène qui anime en banlieue un petit groupe thérapeutique. L'ethnographe, tombé par hasard sur le prospectus dudit groupe est d'abord intrigué par son programme : il est destiné aux Aborigènes (bien qu'il se présente formellement comme ouvert à tous) souhaitant opérer des changements dans leur vie. K propose aux individus intéressés de se réunir une fois par semaine pendant deux heures afin d'échanger sur leurs histoires respectives. Il propose ainsi un espace au sein duquel, en s'appuyant sur des techniques qui ont pour but de réduire le stress et la colère, les participants communiquent, partagent leurs expériences de vie et entament un " processus thérapeutique ». L'objectif déclaré du programme consiste à «briser les schèmes des comportements problématiques » (c'est ce que lit l'ethnographe sur le prospectus) qui, depuis plusieurs générations, rongeraient les communautés aborigènes du pays, alimentant les polémiques concernant les politiques publiques qui leur sont destinées.

A priori, ces séances n'intéressent que très moyennement l'ethnographe, puisqu'elles ne semblent pas concerner directement son sujet d'étude : le traumatisme colonial stricto sensu ne semble pas y tenir une place centrale. Simplement, il est curieux d'en apprendre davantage sur les motivations de son organisateur et espère secrètement que $\mathrm{K}$, puisqu'il travaille dans le domaine thérapeutique, pourra le rediriger vers une personne ressource ou une association traitant directement de la problématique qui l'intéresse. Il le contacte donc par e-mail, lui explique brièvement le contenu de ses recherches et lui demande s'il serait possible qu'ils se rencontrent et discutent quelque peu, «casually», de ses occupations. K accepte et lui fixe un rendez-vous le lundi soir à 18 heures, dans la cafétéria du centre omnisport dans lequel il s'entraîne régulièrement. Jusque là, les choses sont claires pour l'ethnographe : il a rendez-vous avec K pour discuter avec lui de ses activités en lien avec le groupe d'entraide qu'il anime. Il s'attend ainsi à faire la connaissance de $\mathrm{K}$, espère qu'entre eux le courant passera suffisamment bien pour que ce dernier lui fournisse de « bonnes adresses » ainsi que des conseils pour la suite de son enquête. L'ethnographe, «préparé » à la surprise (souvenez-vous), n'est pas dérouté par ce lieu de rencontre pour le moins singulier.

Le jour $\mathrm{J}$, après s'être égaré quelque peu dans les ruelles du quartier, il arrive au lieu dit avec trente minutes d'avance. Il constate que le centre omnisport est situé dans un ensemble plus vaste, une sorte de complexe hôtelier consacré, semble-t-il, aux Aborigènes ${ }^{2}$. Un petit passage par la réception lui apprend que de nombreuses

2 En réponse aux nombreux rapports gouvernementaux et non-gouvernementaux produits sur la question du gouffre catastrophique (dans les domaines les plus variés : santé physique, santé mentale, éducation, accès aux ressources, emploi, etc.) existant entre les Australiens d'origine indigène (« blackfullas ») et les autres ("whitefullas »), plusieurs solutions visant à réduire cet écart ( close the gap »), ont été proposées et financées par le gouvernement australien. Parmi ces dernières, la construction de centres omnisports destinés aux Aborigènes rencontre un certain succès, particulièrement dans les quartiers à forte concentration indigène. Ces centres ont pour ambition d'encourager la pratique du sport et ainsi de sensibiliser les personnes concernées à la question de la santé physique et mentale. 
salles sont destinées à la location pour des événements de tous ordres, que l'on peut également louer des chambres à coucher, faire appel à un service traiteur, etc. Intrigué mais toujours pas surpris - pas encore -, l'ethnographe demande le chemin du centre omnisport. On le lui indique et il se met en route d'un pas quelque peu hésitant. Il entre dans le hall et, s'adressant au réceptionniste, demande à voir $\mathrm{K}$. " C'est pour le groupe ? » lui demande-t-on. Quelque peu désorienté et ne sachant pas trop quoi répondre, l'ethnographe acquiesce. On lui indique alors que K n'a pas encore terminé sa séance d'entraînement, mais qu'il ne devrait plus tarder. On lui propose de prendre une chaise et de patienter près des tables de la cafétéria. L'ethnographe s'exécute et s'assied. Il prend alors conscience du monde autour de lui : des bruits de fonte soulevée avec effort et relâchée avec satisfaction, de la musique pop envahissante ; il constate l'agitation ambiante. Tous les individus présents semblent savoir pourquoi ils sont là et où ils doivent se rendre. La plupart d'entre eux paraissent aborigènes.

Pour se donner une contenance, l'ethnographe s'empare du journal local qui traîne sur le rebord du comptoir, car, ce qu'il n'ose pas encore s'avouer en cet instant précis, c'est qu'il ressent une anxiété croissante : il était venu pour discuter brièvement avec $\mathrm{K}$ et s'attendait à ce que cette entrevue soit des plus anodines. Or, les lieux ne semblent pas adéquats pour une telle rencontre. Qui plus est, il commence à se sentir quelque peu voyeur, étranger même, à regarder ici et là, dans ce centre omnisport destiné avant tout à l'activité physique. Nerveusement, il tourne les pages de son journal sans vraiment rien lire. Plutôt, il observe et écoute ; il cherche à comprendre où il est et à savoir ce qu'il fait là. L'angoisse monte et $\mathrm{K}$ n'arrive toujours pas. Il songe alors à partir, à reprendre contact avec $\mathrm{K}$ et lui présenter ses excuses par e-mail pour ne pas avoir pu être là ce soir, lorsqu'un individu au profil décharné s'approche. L'homme, d'une cinquantaine d'années, s'assied en face de lui et lui demande si c'est sa première fois. Ebahi, l'ethnographe balbutie dans un anglais approximatif qu'il est là pour rencontrer K. L'homme, au cou tatoué et au regard perçant, opine du chef en signe de compréhension et répète sa question : « et c'est la première fois, alors ? ». «La première fois de quoi ? »se demande l'ethnographe. A nouveau, ne sachant pas quoi répondre, il finit par produire un « oui » timide. L'homme appelle alors l'un de ses amis, qui se tenait nerveusement contre la porte d'entrée et que l'ethnographe n'avait pas encore remarqué. L'homme tatoué se présente et, d'un même geste, présente son ami qui était venu s'asseoir à leur table : « mon nom est $\mathrm{P}$ et voici $\mathrm{D}$; lui, c'est sa troisième fois ». L'ethnographe, cherchant à retrouver un minimum de contrôle sur la situation qui lui échappe de plus en plus, demande à ces messieurs s'ils connaissent K. Les deux hommes hochent la tête. $\mathrm{P}$ demande à l'ethnographe d'où il vient et l'échange se poursuit comme cela pendant quelques minutes... lorsque, finalement, $\mathrm{P}$ et $\mathrm{D}$, semblant avoir perçu un certain signal, se lèvent brusquement, se dirigent vers la porte d'entrée et sortent. Confus, surpris, l'ethnographe est plus alerté que jamais et, cherchant à comprendre ce qui se passe, observe avec concentration les mouvements autour de lui. Il remarque qu'un homme grand et très bien bâti vient d'ouvrir une porte renfoncée donnant sur une salle connexe, à côté du comptoir de l'accueil. Le réceptionniste s'adresse à lui et, d'une manière volontairement bruyante (sans doute, pour que l'ethnographe puisse entendre le commentaire) lui dit : « $\mathrm{K}$, le jeune homme blond, là-bas, te demande ». $\mathrm{K}$ détourne à peine les yeux et s'enfonce dans la salle. 
L'ethnographe se lève et le suit, ayant cru comprendre que cet homme musclé n'est autre que $\mathrm{K}$, la raison de sa présence en ces lieux. Et, alors qu'il s'avance, les deux autres hommes qu'il a rencontrés plus tôt - P et $\mathrm{D}-$, les bras chargés de caisses de victuailles, lui coupent le passage et s'infiltrent dans la salle. Juste après, $\mathrm{K}$ en ressort et, s'adressant à l'ethnographe, lui ordonne de porter les caisses à l'intérieur. Plus confus que jamais, il s'exécute. Après quoi, il reste là, debout, à attendre le retour de K. Plusieurs autres personnes s'invitent dans la salle et commencent à s'activer : certains installent des chaises en cercle, d'autres disposent un drapeau aborigène et différents ustensiles au centre du cercle ; d'autres sortent la nourriture (des sandwiches et des fruits) des caisses.

L'ethnographe, l'esprit affûté par l'angoisse, remarque que toutes ces personnes mettent un soin particulier à disposer les objets bien au centre du cercle formé par les chaises. Il remarque encore que l'assemblée est constituée d'une dizaine d'hommes et de femmes de tous âges et, semble-t-il, de tous horizons ; ils rient et, pour la plupart, ont l'air de se connaître. L'ethnographe, lui, se demande ce qu'il fait là.

Quand, finalement, K entre à nouveau dans la pièce, l'ethnographe l'accroche et se présente en chuchotant et bégayant quelque peu. K ne semble pas s'en soucier; il lui dit bonjour et, lui tendant une pile de feuillets, lui demande de les plier en trois afin d'en faire des prospectus. Il lui montre comment faire, puis s'installe sur une chaise. L'ethnographe, assis à une petite table plus loin, observe et écoute ; de plus en plus étonné et de plus en plus anxieux vis-à-vis de la tournure que prennent les événements, il s'exécute en silence. Tout en pliant les feuillets, il remarque que ceux-ci sont fort similaires au programme qu'il avait précédemment obtenu par hasard, au cours de ses errances dans le quartier. Il plie et écoute. Plie et observe. Plie et écoute et finit par entendre $\mathrm{K}$, assis sur sa chaise et s'apprêtant à manger une banane, dire aux autres membres de l'assemblée que l'ethnographe est là pour mieux comprendre ce qu'ils font ici toutes les semaines. "Ah bon ? »s'étonne silencieusement l'ethnographe, surpris par cette dernière remarque, alors que tous se mettent à rire. Plus anxieux et mal à l'aise que jamais, il ne sait plus quoi faire d'autre que de plier ses prospectus, rougir et sourire bêtement.

Enfin arrivé au bout de sa pile, l'ethnographe finit par revenir vers $\mathrm{K}$ et lui demande s'il a bien reçu son e-mail. Son espoir est bien évidemment de rectifier la situation, que comme lui, K se rende compte qu'il y a malentendu, qu'il l'invite à quitter la salle et qu'ils reportent leur rendez-vous à une date ultérieure : au fond, ne devaient-ils pas simplement se rencontrer en tête-à-tête afin de discuter un peu des activités de $\mathrm{K}$ ? Ne devaient-ils pas se retrouver seulement à deux ? Ne devaient-ils pas apprendre à se connaître et - peut-être, mais peut-être seulement - l'ethnographe collecter le nom de l'une ou l'autre personne pouvant lui permettre de l'aider à définir le futur terrain de sa recherche doctorale?

$\mathrm{K}$, le regardant pour la première fois dans les yeux, lui répond « oui, Eddie (c'est le surnom anglo-saxon de l'étranger). Maintenant, assieds-toi, là, à côté de moi, on va commencer. »

La réponse claire et concise de K sidère l'ethnographe. Il s'assied la boule au ventre et se demande ce qui va se passer. En attendant la suite, il s'interroge : comment, ce « oui » est-il possible ? A quoi se raccroche-t-il ? Qu'est-ce que K pose en affirmation? Quelles étaient ses intentions initiales vis-à-vis de lui ? A-t-il oublié leur rendez-vous 
et, ne voulant pas perdre la face devant les autres, fait-il semblant de savoir de quoi il en retourne, ou bien $\mathrm{K}$ avait-il initialement compris que l'ethnographe désirait participer à son groupe de parole ? Et si c'était lui, l'ethnographe, qui avait mal compris ? Et si c'était lui qui, en fixant ce rendez-vous, s'était attendu à quelque chose de précis, de trop précis?

Après quelques secondes, lorsqu'il finit par comprendre qu'il va effectivement participer au groupe de parole, qu'il va en être " de l'intérieur », l'ethnographe s'angoisse plus encore et se dit que, vraiment, entre lui et $\mathrm{K}$, le malentendu est énorme : venu pour un petit entretien innocent, il finit par participer à un processus thérapeutique collectif aborigène, au cœur de la plus grande ville australienne. Comment en est-il arrivé là ?

Après la séance, $\mathrm{K}$ ne fournira pas plus d'explications à l'ethnographe. Il lui demandera de l'aider à charger - en silence - sa voiture des victuailles restantes. Lorsqu'il refermera la porte de son coffre, il déclarera : «Bien, Eddie, à la prochaine... N'oublie pas, c'est tous les lundis de 18 à 20 heures... Salut !»

Et l'ethnographe n'allait pas oublier... En effet, ce groupe de parole est depuis lors devenu l'objet principal de ses recherches doctorales.

\section{Conditions épistémiques et émotionnelles de l'anthropologue en action : pour un rééquilibrage pratique}

Cette vignette ethnographique n'a rien d'exceptionnel. En effet, elle traite du quotidien de l'enquêteur en herbe, de ses rencontres, trébuchements et balbutiements, de ses incompréhensions et questionnements intérieurs, de ses ressentis affectifs. Bref, cette description essaye de rendre dans le détail un moment parmi tant d'autres dans le quotidien du chercheur que je suis. Malgré son apparente simplicité, cette petite tranche de vie peut cependant nous apprendre beaucoup sur la condition épistémique, émotionnelle et pratique de l'anthropologue en action. C'est l'aspect banal des événements traités dans ce court récit qui permettra d'explorer cette condition, de l'exemplifier, de la décortiquer et de comprendre les rapports qu'elle entretient avec les thématiques voisines de l'angoisse et du malentendu. En effet, nous découvrons à la lecture de cette vignette, un ethnographe soucieux, angoissé et affectivement sensible aux événements qui se produisent autour de lui et qui l'incluent. Mais avant d'aller plus loin, demandons-nous d'abord ce qu'est un ethnographe ? Si cette question peut paraître triviale, voire rhétorique, il n'en est rien ; c'est en elle que se cachent la plupart des « non-dits » de l'anthropologie subtilement abordés et traités par Sophie Caratini (2012). Ils nous permettent de toucher de près la réalité de la condition épistémique de l'ethnographe. Cette question est donc un passage obligé.

Si l'ethnographe peut sembler aventureux, téméraire, observateur et empathique, cet individu dont Bronislaw Malinowski pourrait, à première vue, représenter l'idéaltype - nous verrons qu'il n'en est rien -, le profil concret (réel) de l'anthropologue n'est pas toujours aussi simple à croquer. Ou plutôt, il est plus nuancé car c'est généralement a posteriori, lorsqu'il s'agit de rentrer chez lui et de mettre son « aventure » par écrit et entrer dans le discours de la science, qu'il devient cet être lumineux et plein d'assurance. C'est en effet au travers de son discours, de ses explications (textuelles, notamment) et de sa façon de présenter les choses que la figure de l'anthropologue apparaît et se 
réalise (Ghasarian, 1997). Sur le terrain pourtant, comme le montre la précédente vignette, il est bien plus bancal, hésitant, maladroit, hypocrite et anxieux qu'il ne veut généralement l'admettre ${ }^{3}$. Comme le remarque Bruno Latour, « tout chercheur qui a eu à présenter ses recherches à l'extérieur sait très bien la différence abyssale qui sépare la conception que le public se fait de la science faite et le vécu quotidien du chercheur, qui est la réalité de la recherche, c'est-à-dire la science en train de se faire. » (Latour, $2001: 11)$

L'évidence de cette « différence abyssale » apparaît avec force lorsque l'on entame les premières lignes de Tristes Tropiques, là où Claude Lévi-Strauss nous apprend qu'il « hai $[\mathrm{t}]$ les voyages et les explorateurs » $(1955: 11)^{4}$. Ce contraste entre l'anthropologie faite et l'anthropologie en train de se faire apparait avec plus de netteté encore lorsque l'on compare la taciturne neurasthénie qui traverse la totalité du Journal de Bronislaw Malinowski (1985) avec les directives méthodologiques enjouées et systématisées de l'introduction de ses Argonautes (1989). Mais que ces «non-dits » participent pleinement de la condition épistémique et pratique de l'ethnographe n'a rien d'étonnant. En effet, celui-ci doit investir sa personne tout entière dans sa profession. Car, comprendre l'Autre, c'est avant tout « créer des liens » avec ce dernier, s'investir dans son quotidien, s'imprégner de sa vie, ses habitudes, ses schèmes de pensées. Une fois sur le terrain, l'ethnographe doit faire avec ses propres ressentis affectifs. Son corps - ce « sac à surprises » (Pennac, $2012: 12$ ) - devient alors un élément de travail important avec lequel il doit composer (Devereux, 1980 ; Caratini, 1997) puisqu'il s'expose et se rend vulnérable (Behar, 1996) aux aléas de l'enquête. Il s'expose aux dangers physiques du terrain (Howell, 1988) mais surtout aux difficultés émotionnelles et relationnelles qu'il peut y rencontrer (Caratini, 2012 ; Kleinman and Copp, 1993). Si être anthropologue nécessite de voyager et de rencontrer l'Autre, cela ne signifie pas pour autant que tout se passe toujours au mieux avec celui-ci. Parce qu'il ne maîtrise pas complètement les codes du monde social qu'il cherche à comprendre et étudier, l'anthropologue s'expose aux malentendus et se rend donc vulnérable aux angoisses que ceux-ci engendrent. En effet, ces deux expériences que sont l'angoisse et le malentendu, expérience affective pour la première et communicationnelle pour la seconde, prennent leurs racines dans le hiatus interactionnel de la rencontre, dans cette confrontation avec l'Autre dont on ne connait pas (encore) grand-chose.

Observation, participation, communication, toutes ces approches dont s'outille et se sert quotidiennement l'anthropologue pour parvenir à faire son travail, sont autant de moments potentiellement générateurs de malentendus (Servais et Servais, 2009) et d'angoisse (Caratini, 2012). L'interaction est trop peu maîtrisée par ce dernier pour pouvoir se dérouler de manière prévisible, sans confusion; de ce fait, elle peut engendrer le malentendu. C'est ainsi que dans cette première vignette, le jeune ethnographe que je suis enchaîne les (et s'enchaîne aux) malentendus jusqu'à se retrouver dans une situation complètement inattendue. A cet égard, notons d'ores et déjà à quel point le contrôle de la situation (aussi bien d'ailleurs que le contrôle de soi) peut lui échapper. « Pris », englué dans l'action, il manque du recul nécessaire que demande toute saisie

3 Pour d'autres exemples, voir Barley, 2001 ; Behar, 1996 ; Caratini, 2012 ; Favret-Saada, 1977 ; Rabinow, 1977 ; Wacquant, 2002.

4 Ouvrage dont la réception fut, par ailleurs, mitigée dans la communauté des anthropologues. 
« stratégique » de la situation. Il n'a d'autre choix, pour reprendre les catégories de l'action de Michel de Certeau (1990), que de recourir à des « tactiques » et, a fortiori, de faire preuve d'adaptation, de souplesse pratique. Il se doit d'improviser et de s'accommoder des éléments qu'il rencontre. Or, plonger si mal armé dans un univers aussi instable engendre nécessairement de l'angoisse.

Mais la vulnérabilité de l'anthropologue ne s'arrête pas là. Car, au-delà du moment de l'action, être anthropologue, c'est également douter quotidiennement de ses capacités de compréhension et d'observation, c'est souffrir de la distance d'avec sa famille et ses amis, c'est appréhender la retranscription de l'expérience vécue (Behar, 1996 ; Caratini, 2012 ; Kleinman et Copp, 1993). Bref, faire de l'anthropologie et être anthropologue, c'est être en proie à des émotions parfois difficiles à maîtriser, parfois complexes à expliciter et à reconnaître, c'est lutter sans cesse contre le « déracinement chronique » (Lévi-Strauss, 1955), contre le stress et la fatigue, c'est être un " animal hybride ", errant à la frontière de plusieurs mondes (Schutz, 2003), c'est appréhender le danger et l'expérience du terrain (Powdermaker, 1966 ; Wacquant, 2002) et se confronter aux difficultés qui peuvent naître dans l'interaction de la rencontre. Etre anthropologue et faire de l'anthropologie, c'est donc aussi ressentir l'affect de l'angoisse et faire l'expérience du malentendu.

Cela étant, comment continuer à espérer réaliser un travail anthropologique de qualité si le portrait de l'anthropologue en action que je viens d'esquisser est bel et bien fondé sur une condition épistémique, émotionnelle et pratique que l'on pourrait qualifier de « fragile », de « vulnérable »?

Avant de tenter d'apporter des pistes de réponses à cette question épineuse, notons encore qu'en concevant le travail de terrain davantage comme un corpus de « stratégies » qu'il suffirait de mettre en œuvre plutôt que comme une série de « tactiques » nécessitant inventivité et adaptation aux conditions (affectives et interactionnelles, notamment) de l'enquête, les manuels de méthodologie tendent à transformer cette fragilité épistémique en un problème moral. En effet, à leur lecture, le « terrain » semble se résumer à toute une série de difficultés pratiques et théoriques qu'une bonne préparation en amont et une bonne organisation permettraient de résoudre (Beaud et Weber, 2003 ; Mauss, 1989 ; Weber, 2009). L'anthropologue qui rencontrerait malgré tout des difficultés à réaliser son travail pourrait être alors considéré comme un " mauvais anthropologue », c'est-à-dire comme ne s'étant pas suffisamment préparé au terrain et/ou ne connaissant pas suffisamment la « théorie de la méthode » anthropologique pour « bien » réaliser son métier.

Plutôt que de considérer cette condition épistémique comme un problème moral, il est plus fécond de l'aborder comme une problématique méthodologique. Ce faisant, il ne s'agit plus tant d'adopter un point de vue moral (« est-ce bien ou mal de ressentir de l'anxiété sur son terrain ? », « le malentendu est-il une faute de la part du pratiquant en anthropologie ? »), que de comprendre comment, partant de la condition épistémique qui est la sienne, celui-ci parvient pratiquement à faire de l'anthropologie. Evitant dès lors de percevoir les angoisses et les malentendus - inhérents à la pratique - comme des obstacles méthodologiques qu'il faudrait à tout prix éviter, il s'agirait plutôt de chercher à démontrer en quoi et comment ces expériences permettent à l'anthropologue de réaliser son métier (Devereux, 1980). En s'interdisant de considérer cette condition épistémique de l'anthropologue comme un problème et en l'abordant comme une 
problématique, on s'autorise l'analyse rigoureuse des processus cognitivo-affectifs qui entrent en jeu dans la réalisation de l'expérience ethnographique. Ce faisant, on se permet d'en comprendre les ressorts et le fonctionnement dans l'action et, a fortiori, l'influence de ces derniers dans la construction du savoir anthropologique.

\section{Les aspects positifs de l'étonnement, de l'angoisse et du malentendu}

Revenons un instant sur mon expérience afin de chercher à comprendre et à analyser le déroulement des événements lors de cette première rencontre avec $\mathrm{K}$ et son groupe. Ce faisant, j'espère extraire de cette analyse des intuitions théoriques me permettant de démontrer l'intérêt méthodologique du malentendu et de l'angoisse qu'il suscite pour la pratique de l'anthropologie.

Dans cette première vignette, ce que l'on constate avant tout, c'est que je me fais surprendre : je suis bel et bien «pris » dans le terrain mais d'une manière différente de celle à laquelle je m'attendais. Malgré une préparation au terrain particulièrement minutieuse, je ne peux éviter le choc interrogatif et l'incompréhension générale de la situation donnée. Mon intention initiale était de rencontrer $\mathrm{K}$ afin que celui-ci puisse me rediriger vers quelqu'un d'autre, quelqu'un s'inscrivant plus spécifiquement dans le cadre de mes recherches. Visiblement, si à première vue, $\mathrm{K}$ avait bien compris mes attentes (en répondant favorablement à mon e-mail et en me donnant un rendez-vous), au fil du temps, les nœuds communicationnels entre nous se sont multipliés et épaissis. A tel point qu'à la fin de l'épisode relaté dans la vignette, je ne sais même plus à quoi son affirmation fait référence. Ainsi, je perds le contrôle de la situation (si tant est que je l'ai eu auparavant) et dois me montrer bon tacticien, bricoler des solutions interactionnelles, improviser la plupart de mes comportements en les adaptant aux circonstances.

Outre son aspect comique, ce que la situation illustre avant tout, c'est que le quotidien de l'ethnographe est fait de contingences. En effet, celui-ci cherche et, en toute logique, ne sait pas ce qu'il va trouver. Proposition triviale mais, une nouvelle fois, riche en enseignement. Car, la surprise n'est pas seulement le produit du hasard ; elle doit être recherchée, pistée, traquée par le chercheur. Celui-ci doit en effet être surpris pour pouvoir « y être pris » et, plus tard, se « reprendre » (Favret-Saada, 2009). Mais pour que puisse survenir la surprise, cette condition première de la recherche, le chercheur doit avant tout accepter de se laisser faire par le terrain, être étonné par ce dernier, c'est-à-dire s'aménager une posture d'ouverture face à l'inconnue que celui-ci représente. Puisque, comme le précise Chiara Cavalli en se basant sur les réflexions de Martin Heidegger, «l'étonnement ne serait pas seulement l'étincelle allumant l'activité du philosophe, mais bien plus ce qui la soutient et la nourrit d'un bout à l'autre de son déploiement » (2015), se laisser la possibilité d'être étonné sur et par le terrain, est une condition primordiale de la recherche. Or, une fois que la surprise survient, l'ethnographe n'a d'autre choix que d'éprouver quelque chose, que « d'être affecté » (Favret-Saada, 2009). L'y voilà « pris ». Et, parce que dans une telle situation, celui-ci est rendu vulnérable, il éprouve de l'angoisse ${ }^{5}$.

5 Comme le précisait déjà Georges Devereux, « le chercheur est émotionnellement impliqué dans son matériau [...] ; ce qui, en dernière analyse, rend l'angoisse inévitable » (1980:30). 
Comme tout affect, l'angoisse peut être décrite comme la résolution d'une tension entre l'individu et l'environnement : ce sont bien les « affections par les choses extérieures » (Lordon, $2013: 78$ ) qui vont influencer les ressentis corporels et mentaux des individus au moment de l'interaction et provoquer la sensation. Acet égard, l'angoisse - qui, dans mon cas, est engendrée par le décalage entre des attentes (trop) précises quant à ce rendez-vous et la tournure que prennent effectivement les événements - est un affect particulièrement puissant puisque son expression psychophysiologique est importante : " malaise diffus qui sourdement enfle et se propage, irradie et revient par vagues : boule au creux de l'estomac, étreinte qui enserre la gorge, empêche de respirer, palpitations, compression de la cage thoracique, constriction douloureuse des muscles » (Grossman, 2008 : 9). Pour Sigmund Freud, l'angoisse "désigne un état caractérisé par l'attente du danger et la préparation à celui-ci, même s'il est inconnu » (1981 : 50). Pour ce dernier,

l'angoisse est incontestablement en relation avec l'attente; elle est angoisse de quelque chose; elle a pour caractères inhérents l'indétermination et l'absence d'objet; dans l'usage correct de la langue son nom lui-même change, lorsqu'elle a trouvé un objet et il est remplacé par celui de peur. (Freud, 1951 : 94)

Eprouver de l'angoisse, c'est donc redouter quelque chose d'incertain, d'indéfini, de flottant. En fait, l'angoisse, c'est la peur d'avoir peur. C'est la peur de souffrir encore. C'est une posture face à l'inconnu, une « disposition face au néant » (Heidegger, cité par J. de Gramont, 1991 : 132). Compris en ce sens, l'étonnement et l'angoisse sont donc liés l'un à l'autre : ce sont deux postures distinctes et pourtant conjointes (l'angoisse succédant à l'étonnement) d'ouverture face à l'ignorance, deux dispositions face à l'inconnu. Malgré son aspect émotionnellement désagréable, l'angoisse est donc bien un indice de l'étonnement que provoque en nous cette étrangeté, cet inconnu, cet Autre et doit être interprétée comme un signe d'ouverture à celui-ci. L'angoisse peut donc servir l'anthropologue en devenant pour ce dernier un outil précieux - par l'effet direct qu'elle exerce sur le corps -, un indice de surprise et donc de " prise », un générateur de questionnement et, in fine, de découverte. Elle peut même mener l'anthropologue jusqu'au décalage créatif: ce décalage qui, provoqué par l'étonnement, « jette l'homme hors de l'engagement de la familiarité quotidienne avec l'étant [...] vers l'indigence créatrice d'un non-savoir de l'être de l'étant " (Fink, 1974 : 203). Autrement dit, l'angoisse peut permettre phénoménologiquement de décaler, créant ainsi chez celui qui l'éprouve, un regard « autre », « neuf » sur les phénomènes qui l'entourent ${ }^{6}$.

Bien sûr, cette angoisse ne vient pas de nulle part. Elle advient lorsque l'individu se retrouve dans la situation de vulnérabilité où l'a placé le quiproquo ou le malentendu. $\mathrm{Si}$, dans la vignette qui nous occupe, je commence à ressentir de l'angoisse, c'est avant tout parce que $\mathrm{K}$ n'arrive pas et que, petit à petit, la confusion engendrée par le malentendu initial concernant le but de notre rencontre s'installe dans mon esprit. Progressivement, je réalise que la situation dans laquelle je me trouve effectivement ne correspond pas à celle que $\mathrm{j}$ 'avais imaginée. Ainsi, au fur et à mesure que l'action se déroule, je suis de plus en plus étonné par ce qui se passe autour de moi : le décalage entre mes attentes et la réalité s'agrandit, le malentendu s'épaissit et, avec lui, l'angoisse

6 Voir, à titre d'exemple, la seconde vignette ethnographique. 
s'accentue. Or, comme l'ont montré plusieurs auteurs (Colombetti et Krueger, 2015), l'état émotionnel d'un individu est directement influencé par la conception qu'il s'en fait. Pour le dire autrement, un état affectif restera à l'état de «background feeling » (Damasio, 1999) ${ }^{7}$ tant qu'il n'est pas exprimé d'une manière ou d'une autre, fût-ce sans l'extérioriser. L'absence de formulation condamne ce ressenti affectif situé à la périphérie de la conscience ${ }^{8}$ à rester indéfini et, par conséquent, à ne pas être totalement expérimenté. Pour être pleinement vécue, une émotion doit être exprimée, et c'est bien cela que je fais - exprimer mon angoisse - lorsque, à plusieurs reprises, je m'interroge sur les raisons de ma présence dans ce centre omnisport, m'empare d'un journal, plie mes feuillets pour « faire quelque chose » et lutter contre l'attente et l'indétermination.

Ainsi, comme cette courte vignette le montre, le malentendu et son lot de surprises, d'étonnement et d'angoisse caractérisent l'entreprise anthropologique. Sur le terrain, la plupart du temps solitaire, l'anthropologue doit composer quotidiennement avec des éléments contingents. Ces derniers proviennent à la fois de l'environnement extérieur (rencontres, lieux, événements) et de l'intérieur (émotions, pensées, imaginations, sensations, impressions). Face à cette situation, le premier geste épistémique et méthodologique tendrait, presque instinctivement, à supprimer ces impondérables, ces hiatus, cette contingence du travail scientifique au profit d'une méthode d'enquête plus lissée, plus « neutre », plus maîtrisée. Mais ce mouvement induirait deux erreurs majeures. Premièrement, la confusion entre théorie et pratique : la contingence ne peut être évitée, repoussée pratiquement, puisqu'elle constitue la condition même de la recherche et de la vie quotidienne du chercheur. Deuxièmement, elle aboutirait à priver le chercheur des avantages (conséquents) d'une posture scientifique vulnérable mais efficace, car assumée. Comme le précisait George Devereux, « l'important n'est donc pas de savoir si on utilise la méthodologie aussi comme un moyen de réduire l'angoisse, mais de savoir si on le fait en connaissance de cause, de manière sublimatoire ou, de façon inconsciente, seulement de manière défensive. » (Devereux, 1980 : 148)

\section{Vignette ethnographique II}

Me voici donc participant à cette séance de thérapie collective. Inutile de rappeler mon niveau d'anxiété élevé face à cette situation nouvelle, surprenante et déstabilisante... Assis sur l'une des chaises en plastique disposées en cercle autour d'un drapeau aborigène et d'autres petits objets à fortes connotations symboliques et culturelles, j'observe et écoute. Je constate la présence d'une petite pile de feuillets à ma gauche ; je remarque la mine grave de certains individus, leur nervosité apparente. Je note aussi la posture plus décontractée d'autres participants. En captant ça et là des

7 Antonio Damasio entend par «background feelings » les ressentis somatiques nébuleux et indéfinis qui ne sont pas des sensations corporelles spécifiques mais qui sont plutôt des façons de faire l'expérience physique du monde (Colombetti, $2009: 7)$.

8 Pour Mikhaïl Bakhtine, « la pensée n'existe pas en dehors de son expression potentielle et par conséquent en dehors de l'orientation sociale de cette expression et de la pensée elle-même. [...] En dehors de son objectivation, de sa réalisation dans un matériau déterminé (le geste, la parole, le cri) la conscience est une fiction. [...] Mais, en tant qu'expression matérielle structurée (à l'aide du mot, du signe, du croquis, de la peinture, du son musical, etc.), la conscience constitue un fait objectif et une force sociale immense » (italiques de l'auteur) (Bakhtine, 1977 : 129). 
bribes de conversations, je comprends que ces derniers - les plus décontractés - sont des habitués du groupe de K. Je ne sais pas véritablement à quoi m'en tenir puisque, ne m'attendant pas à participer à une telle séance, je ne me suis pas renseigné sur ce qu'auraient pu être son déroulement et son contenu.

Au bout de quelques minutes, $\mathrm{K}$ prend la parole et demande aux autres intervenants s'ils ont «quelque chose à raconter ». Les uns après les autres, les divers membres du groupe vont prendre la parole et commencer à raconter leurs « difficultés » de la semaine : pour certains, ne pas boire d'alcool fut particulièrement compliqué car la tentation était grande ; pour d'autres, la semaine s'était plutôt bien déroulée et ils en sont relativement satisfaits. D'autres encore expliquent qu'ils ont « exécuté leur tâche de la semaine » avec brio, etc.

Perdu, car je ne comprends pas la signification de ces échanges langagiers, je vais alors tendre l'oreille, redoubler d'attention et chercher à comprendre le contexte pragmatique dans lequel les mots sont prononcés. Petit à petit, je vais comprendre que ces individus sont venus là pour échanger et parler de leurs expériences douloureuses ainsi que de leurs petites victoires quotidiennes face à l'adversité. Anciens alcooliques, colériques repentis, victimes de violences familiales et conjugales se côtoient et communiquent, partagent leurs histoires et leurs solutions. Je remarque alors que la parole passe de l'un à l'autre, équitablement répartie, et que les histoires se succèdent ; les commentaires sont volontairement positifs et encourageants. Bonne humeur et gravité (de circonstance) cohabitent ; tous passent d'une attitude à l'autre. L'écoute se veut attentive et introspective, chacun se nourrit des expériences des autres, rapportant ces dernières à sa propre histoire, $\mathrm{y}$ cherchant les points de convergence et les attitudes et comportements similaires. Particulièrement attentif aux événements, je note encore avec quel tact et quelle finesse rhétorique $\mathrm{K}$ distribue et coordonne la parole. Sous son impulsion, la plupart des histoires individuelles finissent par emporter l'assentiment généralisé ; la collectivité se crée. Bien que les histoires de chacun soient fondamentalement différentes, tous semblent partager un même affect composite suffisamment puissant pour les réunir : il s'agit tout à la fois d'être liés de près ou de loin à l'identité aborigène ${ }^{9}$, d'avoir connu des difficultés relativement importantes au cours de leur vie et de comprendre la source de ces complications comme découlant directement du « poids de l'histoire ${ }^{10}$ ", de vouloir « conjurer » cette histoire, en sortir, « en finir » avec le traumatisme colonial et sa reproduction. Cela non plus ne m'échappe pas (après tout, c'est sur ce point précis que je travaille). J'enregistre tout cela dans un coin de ma tête. Pour l'instant, j'emmagasine car, toujours anxieux et confus, je me demande si je vais moi aussi devoir m'exprimer. Aux aguets, je patiente, écoute et observe attentivement.

Dans un moment de flottement, $\mathrm{K}$ se tourne subitement vers moi et me demande de choisir un des feuillets, celui que je préfère. Stressé, je me saisis du premier de la pile. K

9 La plupart des participants se présenteront au groupe comme étant Aborigènes ou descendants plus éloignés d'Aborigènes.

10 Plusieurs études scientifiques et rapports gouvernementaux ont montré l'importance de l'histoire coloniale dans la reproduction de certains comportements dysfonctionnels menant au « traumatisme intergénérationnel »(Atkinson, 2002 ; Lavarch, 1997). Ces différentes productions savantes sont généralement bien connues des populations aborigènes urbaines. 
me demande d'en lire le titre à voix haute. Il en profite pour me présenter une nouvelle fois à l'assemblée. Embarrassé, je ne peux m'empêcher de rougir. Je m'exécute et lis le titre du document : « le petit garçon qui ne voulait plus qu'on le frappe ». Ensuite, K me demande de lire le petit texte (d'une quinzaine de lignes) en dessous du titre, toujours à voix haute. Je m'applique. K me remercie et demande alors à l'assemblée ce que ce texte évoque pour eux. Les échanges langagiers reprennent de plus belle. On me demandera même mon avis. Grâce à une écoute attentive et une attention soutenue, je comprends mieux maintenant le principe et le fonctionnement de ce groupe thérapeutique, et je livrerai donc au groupe un bref morceau de ma propre histoire en essayant de la faire correspondre au récit que je viens de lire.

Plus tard, K annoncera à l'assemblée qu'il est temps « d'évacuer les mauvaises énergies en pratiquant le dadirri $\gg$. Ne sachant pas ce que cela veut dire, j'observe, écoute et tente de saisir les moindres faits et gestes des membres du groupe. Tous se mettent à se frotter les mains avec vigueur, à poser ces dernières sur leurs visages respectifs puis, à expirer lourdement. Ensuite, ils gardent le silence. Je copie tant bien que mal le comportement de mes compères. $\mathrm{K}$ finit par briser le silence qui s'est installé et relance les échanges verbaux autour du « devoir » de chacun. L'oreille tendue, je parviens finalement à comprendre que chacun doit se donner une tâche bénéfique, réaliste et réalisable qu'il devra accomplir pour la semaine prochaine. Certains évoquent l'idée de faire plus de sport, d'autres de reprendre contact avec leurs frères et sœurs.

La séance se termine et, chacun en profite pour attraper un sandwich ou un fruit, tout en rangeant le matériel. Embarrassé, je ne sais que faire et continue d'observer et d'écouter. Je finirai par aider $\mathrm{K}$ à charger sa voiture.

\section{L'éducation de l'attention par le malentendu et l'angoisse}

«Le diable est dans les détails », déclarait Friedrich Nietzsche. C'est très certainement exact... mais, pour le sociologue, ce qui se cache dans les détails, c'est avant tout le sens et, avec lui, la compréhension du social. A cet égard, Erwing Goffman a démontré tout au long de son œuvre l'importance des «petits riens » dans la construction du sens social (Goffman, 1974 ; Goffman, 1987). Doué d'une faculté d'observation particulièrement affutée, il est parvenu à mettre en évidence le fait que la plupart des regards, gestes, postures et énoncés verbaux émis durant l'interaction sont autant d'indices qui permettent de donner un sens aux actes manifestés dans l'interaction. Ce faisant, c'est principalement au travers de cette « micrologie $»^{11}$ (Laplantine, 2003), de cette suite continue de fragments d'interactions, que se joue le sens que les acteurs donnent à leurs actions ; c'est au travers de celle-ci que se dessine le cadre et donc l'enjeu de l'interaction. Par conséquent, c'est également là que se joue - littéralement le social :

Lorsqu'un individu en présence d'autrui répond à un événement, les coups d'œil qu'il lance, ses regards, ses changements de position sont porteurs de toutes sortes de significations, implicites et explicites. Et si des mots sont prononcés,

11 Pour François Laplantine, c'est avant tout dans le minuscule (le peu, le pas, le mien) que se constitue le sens social. D'où l'importance de se pencher sur la logique des micros-interactions - la « micrologie » (Laplantine, 2003). 
le ton de la voix, la manière de la reprise, les redémarrages, la localisation des pauses, tout cela compte de la même façon. Et de même la manière d'écouter. [...] Il y a là une ressource gestuelle qui est partout et constamment exploitée, qui n'est pourtant que rarement examinée de façon systématique. (Goffman 1987 : 8)

C'est cette évidence du tout petit, du presque rien, du détail « micrologique » que j'ai « découvert » lors de ma participation au groupe de parole organisé par K : par divers jeux de langage, stratégies rhétoriques et détails interactionnels, un collectif se crée et la généalogie du traumatisme colonial se (re)construit dans le discours, permettant ainsi à chacun de continuer à donner du sens à ses actes et son existence (la plupart du temps, socialement et économiquement difficile à vivre). Mais pour pouvoir la « découvrir », il m'a d'abord fallu « subir » la situation. En effet, d'abord étonné, puis alerté car angoissé, je vais voir petit à petit mes capacités d'observation et d'attention s'aiguiser ; " mal-entendre ", m'oblige à tendre l'oreille afin de « mieux entendre » : à plusieurs reprises, je devrai donc redoubler d'attention (aux détails) pour pouvoir comprendre l'enjeu - dans le sens littéral de ce qui est en jeu - du groupe de parole et de tous ces échanges langagiers. Ainsi, comme le précise Paul Watzlawick, une fois passé le désarroi initial, le malentendu

\begin{abstract}
déclenche une recherche immédiate de la signification, afin de diminuer l'angoisse inhérente à toute situation incertaine. Il en résulte un accroissement inhabituel de l'attention, doublé d'une promptitude à établir des relations causales [...]. Si cette recherche peut [déboucher] sur un malentendu encore plus grand, elle peut tout aussi bien conduire à des façons neuves et créatrices de conceptualiser la réalité. (Watzlawick, $2014: 35)^{12}$
\end{abstract}

Ce sont sans doute ces capacités qui sont les plus utiles à l'ethnographe. En effet, en augmentant la capacité d'observation et d'écoute de ce dernier, l'angoisse ressentie en situation de malentendu stimule abondamment ses processus cognitifs d'attention, lui permettant de percevoir certains détails interactionnels signifiants qu'il n'aurait pas discernés autrement, et d'éduquer son attention générale à la culture dans laquelle il travaille et qu'il tente de comprendre.

Si l'on revient quelques instants sur la deuxième vignette ethnographique, on pourra remarquer que c'est bien grâce à cette attention redoublée que je parviens à percevoir plusieurs détails microsociologiques d'importance : les jeux langagiers et les stratégies rhétoriques s'y rapportant, la disposition spatiale des lieux produisant un effet fédérateur et égalitaire autour d'un symbole affectif fort (le drapeau aborigène), l'utilisation de la technique du dadirri qui, traditionnellement attribuée aux Aborigènes de Daly River ${ }^{13}$, consiste en une posture d'écoute attentive et d'apprentissage par cette écoute ${ }^{14}$, etc. J'ai été capable d'observer, de mémoriser et de réagencer toute une série d'éléments aussi bien interactionnels que culturels grâce à l'augmentation momentanée de mes capacités cognitives et l'hypersensibilisation de mes capacités affectives ; toutes deux rendues

12 C'est moi qui souligne.

13 Village situé au bord de la rivière du même nom dans le Territoire du Nord (NT).

14 Pour plus de précisions sur le dadirri, se référer à l'article de Miriam Rose Ungunmerr-Bauman (1993) et au livre de Judy Atkinson (2002). 
possibles par le malentendu initial avec $\mathrm{K}^{15}$ et le besoin de décharger l'angoisse qu'il avait engendrée par ce malentendu.

Ainsi, au fil de la séance, mon attention s'est « éduquée »(Gibson, 1979 : 254); dans le flux initialement incompréhensible des éléments m'entourant, déferlant sur moi à toute allure, un ordre, une cohérence interne se sont petit à petit dessinés et se sont laissés « découvrir ». Une signification a pu apparaître au fil de l'éducation de mon attention, de la prise en compte et de la sélection de certains éléments considérés comme porteurs de sens au détriment d'autres. Si je n'avais pas été angoissé, cette signification m'aurait sans doute échappé.

Pour résumer, nous pouvons dire qu'en multipliant les capacités cognitivoaffectives du chercheur, le malentendu et l'angoisse qu'il génère lui permettent d'éduquer son attention, de prolonger l'effort de la concentration et, in fine, d'être plus sensible aux détails et aux subtilités de la vie sociale. Par conséquent, les ambiguïtés communicationnelles du malentendu et les difficultés affectives de l'angoisse que celuici engendre peuvent jouer un rôle pratique important pour le chercheur; d'abord étonné et puis ensuite dérouté, confus, mal à l'aise, ce dernier se doit d'être extrêmement attentif à la situation dans laquelle il est englué pour parvenir à la comprendre et réduire son niveau d'angoisse.

\section{Quelques considérations supplémentaires et conclusion}

«J'ai voulu éclairer la part de subjectivité inhérente à l'expérience anthropologique non pas pour m'en affliger, mais pour montrer que c'est justement elle qui fait toute la richesse de notre discipline. "

Sophie Caratini, Les non-dits de l'anthropologie.

Il n'est intéressant de considérer le malentendu et l'angoisse comme des atouts méthodologiques pour la recherche de terrain que si l'on nuance le propos. En effet, il ne s'agit pas ici de réaliser une apologie de la névrose ; l'angoisse peut, lorsqu'elle est récurrente, se généraliser et devenir un véritable problème de santé mentale avec toutes les complications qu'on lui connaît (Freud 1951). De même, le malentendu peut, s'il est trop profond ou affectivement surinvesti, déboucher sur le conflit ouvert ou la confusion totale. Rien de très constructif, donc, dans ces cas-là... Mais la plupart du temps, subtilement exploitée, l'ambiguïté propre au malentendu et à l'angoisse engendrée par celui-ci peut permettre à l'ethnographe sur son terrain de réaliser pratiquement son métier. La difficulté méthodologique et intellectuelle d'une telle proposition réside peut-être dans la subtile complexité de cette souplesse pratique dont l'ethnographe doit faire preuve face à la contingence. En effet, si l'on ne peut se préparer au malentendu et à l'angoisse auxquels on sera nécessairement confronté lors de la pratique du terrain, on peut néanmoins s'en servir lorsque, finalement, ces expériences surviennent. Et elles surviendront. Par conséquent, rien ne sert de se préparer à être surpris (souvenons-nous d'une part des mots de Emmanuel Belin et, d'autre part, de la naïveté initiale du jeune chercheur que je suis). Cependant, accueillir la surprise, s'aménager la possibilité d'être étonné, pourrait être une sortie élégante du paradoxe. De même, il conviendra de ne pas nécessairement chercher à éviter le malentendu (sans pour autant le provoquer non

15 Voir supra, vignette ethnographique I. 
plus), de s'en accommoder lorsque celui-ci survient et de privilégier la « tactique » (de Certeau, 1990), la souplesse pratique, face à ce dernier. On peut donc utiliser l'angoisse dans ce qu'elle a de positif et non pas chercher à couper court instantanément à une situation jugée anxiogène parce que celle-ci peut apparaitre comme " désagréable ". Solutions élégantes certes, mais surtout efficaces. En effet, comme le précise George Devereux,

\begin{abstract}
les «perturbations » dues à l'existence des activités de l'observateur, lorsqu'elles sont correctement exploitées, sont les pierres angulaires d'une science du comportement authentiquement scientifique et non - comme on le croit couramment - un fâcheux contretemps dont la meilleure façon de se débarrasser est de l'escamoter. (Devereux, $1980: 83$ )
\end{abstract}

Pour ce qui est de mon cas, je n'avais pas comme intention initiale de participer ni encore moins de faire de ce groupe thérapeutique aborigène l'objet principal de mon enquête ethnographique. Or, malgré toute l'angoisse qu'elle a générée en moi, cette rencontre imprévue car issue d'un malentendu, m'a clairement permis d'entamer concrètement mes recherches ethnographiques.

Plutôt donc que de continuer à adopter une attitude visant à réduire - voire carrément supprimer - les contingences de la pratique ethnographique, peut-être pourrait-on exploiter à notre avantage (méthodologique), les inévitables aléas du terrain. Peutêtre devrions-nous accepter l'angoisse, l'utiliser subtilement et saisir les opportunités qu'offrent les malentendus. Se protéger contre les difficultés communicationnelles (le malentendu) et affectives (l'angoisse) du terrain et ainsi sauvegarder la neutralité axiologique du travail accompli, n'est sans doute pas la meilleure manière de résoudre le paradoxe pratique auquel est confronté l'anthropologue sur le terrain. Assumer sa fragilité épistémique et l'exploiter, voilà peut-être le secret. C'est en tout cas le pari que je fais car, me semble-t-il, c'est la seule et unique manière de faire de l'anthropologie et d'être un anthropologue vulnérable mais efficace.

\title{
Références citées
}

AtKinson, Judy, 2002. Trauma trials, recreating song lines : The transgenerational effects of trauma in indigenous Australia. Melbourne : Spinifex Press.

BAKHTINE, Mikhaïl, 1977. Le marxisme et la philosophie du langage : essai d'application de la méthode sociologique en linguistique. Paris : Editions de Minuit.

BARLey, Nigel, 2001. Un anthropologue en déroute. Paris : Payot.

BEAUd, Stéphane et Florence WeBER, 2003. Guide de l'enquête de terrain : produire et analyser des données ethnographiques. Paris : La Découverte.

Behar, Ruth, 1996. The vulnerable observer : Anthropology that breaks your heart. Boston : Beacon Press.

Belin, Emmanuel, 2002. Une sociologie des espaces potentiels : logique dispositive et expérience ordinaire. Paris : DeBoeck Université.

Caratini, Sophie, 1997. « Expérience du terrain, construction du savoir », L’homme, 143, pp. 179-187.

-, 2012. Les non-dits de l'anthropologie; suivi de Dialogue avec Maurice Godelier. Vincennes : T. Marchaisse.

Certeau, Michel de, 1990. L'invention du quotidien. 1, Arts de Faire. Paris : Gallimard. 
Cavalli, Chiara, 2015. "Réflexions sur l'étonnement et l'enseignement de la philosophie au préuniversitaire », Education et socialisation, 39 [En ligne], mis en ligne le 28 septembre 2015, consulté le 29 juin 2016. <http://edso.revues.org/1431>.

Colombetti, Giovanna, 2009. « What language does to feelings », Journal of Consciousness Studies, 16 (9), pp. 4-26.

Colombetti, Giovanna, et Joel Krueger, 2015. "Scaffoldings of the affective mind", Philosophical Psychology, 28 (8), pp. 1157-76.

DAmasio, Antonio, 1999. The feeling of what happens : Body and emotion in the making of consciousness. New York : Harcourt Brace.

De Gramont, Jérôme, 1991. L'entrée en philosophie. Les premiers mots. Paris : L'harmattan.

Devereux, George, 1980. De l'angoisse à la méthode dans les sciences du comportement. Paris : Flammarion.

FaVret-SaAdA, Jeanne, 1977. Les mots, la mort, les sorts. Paris : Gallimard.

-, 2009. Désorceler. Paris : Editions de 1'Olivier.

FInK, Eugen, 1974. De la phénoménologie. Paris : Editions de Minuit.

Freud, Sigmund, 1951. Inhibition, symptôme et angoisse (1926). Paris : PUF.

-, 1981. «Au-delà du principe de plaisir », Essais de psychanalyse (1920). Paris : Petite Bibliothèque Payot.

Ghasarian, Christian, 1997. «Les désarrois de l'ethnographe », L'Homme, 37 (143), pp. 189-98.

GiBson, James, 1979. The ecological approach to visual perception. Boston : Houghton Mifflin.

Goffman, Erving, 1974. Les rites d'interaction. Paris : Editions de Minuit.

-, 1987. Façons de parler. Paris : Editions de Minuit.

Grossman, Evelyne, 2008. L'angoisse de penser. Paris: Editions de Minuit.

Howell, Nancy, 1988. « Health and safety in the fieldwork of North American anthropologists », Current Anthropology, 29 (5), pp. 780-787.

Kleinman, Sherryl, et Martha Copp, 1993. Emotions and fieldwork. Newbury Park/Calif. : Sage Publications.

LaPlantine, François, 2003. De tout petits liens. Paris : Mille et une nuits.

LATOuR, Bruno, (éd.) 2001. Le métier de chercheur, regard d'un anthropologue: une conférence-débat à l'INRA, Paris, le 22 septembre 1994. Paris : Institut national de la recherche agronomique.

LaVArch, Michael (ed.), 1997. Bringing them home : Report of the national enquiry into the separation of aboriginal and torres straight islander children from their families. Sydney : Commonwealth of Australia.

Levi-Strauss, Claude, 1955. Tristes tropiques. Paris : Plon.

Lordon, Frédéric, 2013. La société des affects : pour un structuralisme des passions. Paris : Seuil.

Malinowski, Bronislaw, 1985. Journal d'ethnographe. Paris : Seuil.

-, 1989. Les Argonautes du Pacifique occidental. Paris : Gallimard.

Mauss, Marcel, 1989. Manuel d'ethnographie. Paris : Payot.

Pennac, Daniel, 2012. Journal d'un corps. Paris : Gallimard.

Powdermaker, Hortense, 1966. Stranger and friend: The way of an anthropologist. New York : W.W. Norton.

Rabinow, Paul, 1977. Reflections on fieldwork in Morocco. Berkeley : University of California Press.

SchuTz, Alfred, 2003. L'étranger : un essai de psychologie sociale; suivi de L'homme qui rentre au pays. Paris : Allia. 
Servais, Christine et Véronique Servais, 2009. " Le malentendu comme structure de la communication », Questions de communication, 15, pp. 21-49.

Ungunmerr-Bauman, Miriam Rose, 1993. « Dadirri » in Joan Hendriks et Gerry Hefferan (eds), A spirituality of catholic aborigines and the struggle for justice, pp. 34-37. Brisbane : Aborigines et Torres Strait Islander Apostolate.

WACQUANT, Loïc, 2002. Corps et âme : carnets ethnographiques d'un apprenti boxeur. Marseille : Agone.

Watzlawick, Paul, 2014. La réalité de la réalité : confusion, désinformation, communication. Paris : Points.

WeBER, Florence, 2009. Manuel de l'ethnographe. Paris : PUF. 